\title{
Distribution of class I, III and IV alcohol dehydrogenase mRNAs in the adult rat, mouse and human brain
}

\author{
Dagmar Galter $^{1}$, Andrea Carmine ${ }^{\mathbf{1 , 2}}$, Silvia Buervenich ${ }^{\mathbf{1 , 2}}$, Gregg Duester ${ }^{\mathbf{3}}$ and Lars Olson ${ }^{\mathbf{1}}$ \\ ${ }^{1}$ Department of Neuroscience and ${ }^{2}$ Department of Molecular Medicine, Clinical Neurogenetics Unit, Karolinska Institutet, \\ Stockholm, Sweden; ${ }^{3}$ OncoDevelopmental Biology Program, Burnham Institute, La Jolla, CA, USA
}

The localization of different classes of alcohol dehydrogenases $(\mathrm{ADH})$ in the brain is of great interest because of their role in both ethanol and retinoic acid metabolism. Conflicting data have been reported in the literature. By Northern blot and enzyme activity analyses only class III $\mathrm{ADH}$ has been detected in adult brain specimens, while results from riboprobe in situ hybridization indicate class I as well as class IV ADH expression in different regions of the rat brain. Here we have studied the expression patterns of three ADH classes in adult rat, mouse and human tissues using radioactive oligonucleotide in situ hybridization. Specificity of probes was tested on liver and stomach control tissue, as well as tissue from class IV ADH knock-out mice. Only class III ADH mRNA was found to be expressed in brain tissue of all three investigated species. Particularly high expression levels were found in neurons of the red nucleus in human tissue, while cortical neurons, pyramidal and granule cells of the hippocampus and dopamine neurons of substantia nigra showed moderate expression levels. Purkinje cells of cerebellum were positive for class III ADH mRNA in all species investigated, whereas granular layer neurons were positive only in rodents. The choroid plexus was highly positive for class III ADH, while no specific signal for class I or class IV ADH was detected. Our results thus support the notion that the only ADH expressed in adult mouse, rat and human brain is class III ADH.

Keywords: alcohol dehydrogenase; in situ hybridization; post mortem tissue.
Alcohol dehydrogenases (ADH; EC 1.1.1.1) are among the oldest purified enzymes. All known ADHs are cytosolic, dimeric metalloenzymes composed of about 375 amino acids and a molecular mass of around $40 \mathrm{kDa}$. Each subunit binds two zinc ions, has a binding site for the coenzyme (NADH or NADPH) and a catalytic site. Protein purification and enzymatic studies have led to the identification of different isoenzymes distinguished by substrate specificity and resistance to inhibitors. Relevant to the present study, the class I subunits, ADH alpha, ADH beta and ADH gamma are most active as ethanol dehydrogenases while the class III enzyme is glutathione-dependent formaldehyde dehydrogenase and class IV ADH are the most potent cytosolic retinol dehydrogenases [1].

After the identification of the corresponding genomic sequences, isoenzymes are now grouped according to sequence similarity. In humans, seven different genes are

Correspondence: L. Olson, Department of Neuroscience,

Karolinska Institutet, Retzius väg 8 B2 : 4, 17177 Stockholm,

Sweden. Fax: + 468323 742,

E-mail: lars.olson@neuro.ki.se

Abbreviations: ADH, alcohol dehydrogenase; ADH4-/-, ADH

class IV knock-out mouse; CA, cornu amonis; $\mathrm{CB}$, cerebellum;

gl, granular layer; HC, hippocampus; ml, molecular layer; Pc, Purkinje

cells; SN, substantia nigra; WM, white matter; WT, wild-type.

Enzymes: Alcohol dehydrogenase (EC 1.1.1.1).

(Received 21 November 2002, revised 1 February 2003,

accepted 5 February 2003) known encoding related ADHs, all located in a single cluster on chromosome 4q21-23. The seven genes have been ascribed to five different classes and orthologue genes in rodents and other animals have been found [2]. Amino acid sequence comparisons from multiple vertebrate species indicate that all $\mathrm{ADH}$ classes have evolved from one common ancestor, $\mathrm{ADH}$, presumably class III $\mathrm{ADH}$, the only ADH found also in lower animals, yeast and plants [3]. Table 1 shows the relation between the different ADH genes and proteins and the class-based nomenclature [4]. To simplify the description in different species, we will denominate these genes ADH1, ADH3 and ADH4.

Similar mRNA length and high nucleotide and amino acid sequence identity of all ADHs lead to a large risk for cross-reactivity of probes at the mRNA and protein level, making it difficult to decide which of the ADH genes or proteins is expressed in a certain tissue. In previous studies employing Northern blot analysis, tissue distribution of mRNA for the different ADHs was studied in a variety of species and developmental stages and class III ADH was found to be the only ADH expressed in adult brain $[5,6]$. During development, ADH4 has been shown to be expressed in the floor plate of midbrain by a method making use of a transgenic mouse carrying the ADH4 promoter coupled to a LacZ reporter gene [7].

Because differences in substrate specificity allow a distinction of the enzyme classes in tissue lysates, this function-based method was used predominantly in studies of ADH expressions at the protein level [8]. Such analyses were often focused on the digestive system (liver, stomach, 
Table 1. Alternative names for $A D H$ genes and proteins (in parentheses) within a species and orthologs between the human, rat and mouse $A D H$ genes (based on [4]).

\begin{tabular}{|c|c|c|c|}
\hline \multirow[b]{2}{*}{ Species } & \multicolumn{3}{|l|}{ Abbreviations used in this study } \\
\hline & ADH1 (ADH class I) & ADH3 (ADH class III) & ADH4 (ADH class IV) \\
\hline \multirow[t]{3}{*}{ Human } & $A D H 1 A$ or $A D H 1$ (ADH alpha) & ADH5 (ADH chi) & $A D H 7$ \\
\hline & $A D H 1 B$ or $A D H 2(\mathrm{ADH}$ beta) & Glutathione dependent & (ADH mu or $\mathrm{ADH}$ sigma) \\
\hline & $A D H 1 C$ or $A D H 3$ (ADH gamma) & formaldehyde dehydrogenase & retinol dehydrogenase \\
\hline Rat & Adh1 (Adh1) & $\begin{array}{l}\text { AdhX } \\
\text { (Adh2 or AdhB2) }\end{array}$ & $\operatorname{Adh} 7$ (Adh7) \\
\hline Mouse & $\operatorname{Adh1}(\mathrm{Adh} 1)$ & Adh5 (Adh5) & Adh3 (Adh3) \\
\hline
\end{tabular}

intestine) comparing differences in sex, age or states of disease such as helicobacter infection or gastric ulcer $[9,10]$ with little data on brain tissue available to date.

Cellular localization studies may be more sensitive than tissue-based assays for detection of low expression levels. Thus, in situ hybridization and immunohistochemistry have been performed, again focussing mainly on the digestive tube, excretory, respiratory and sexual systems in different species and developmental stages. Brain tissue has been studied by this methodology predominantly at developmental stages. In one recent study, however, expression of $\mathrm{ADH} 1$ and $\mathrm{ADH} 4$ within distinct cellular populations of adult brain tissue was reported [11]. However, use of partially hydrolyzed ribroprobes in this study may have led to decreased specificity through cross-reactivity with, for example, $\mathrm{ADH} 3$, the 'ancestor' enzyme shown previously to be present in adult brain.

To further investigate the cellular distribution of class I, III and IV ADHs we have carried out in situ hybridization studies in several species using radiolabeled short (49-51 base pairs) oligonucleotides after multiple in silico and in vitro tests for specificity.

\section{Materials and methods}

\section{Animals}

Sprague-Dawley rats (two male and two females, 250$270 \mathrm{~g}$ ) and C57B1/6 mice (two adult males and two adult females, one wild-type and one Adh4 knock-out each [12]) were killed and brains were dissected quickly and flash frozen on dry ice. Similarly, liver and stomach tissue was collected from each of these animals. Stomach samples were rinsed in ice cold phosphate buffer to remove stomach contents before they were flash frozen on dry ice. All samples were kept at $-80{ }^{\circ} \mathrm{C}$ until used. Animal experiments were approved by the Swedish Animal Ethics Committee of Stockholm.

\section{Human tissue}

Human brain tissue was provided by the Harvard Brain Tissue Resource Center (Belmont, MA, USA) and the Netherlands Brain Bank (Amsterdam, The Netherlands). Blocks of cortex, anterior amygdala, striatum and midbrain from four nondemented control subjects (three male and one female, age range (59-79 years), postmortem interval
(PMI) between 4.5 and $23.9 \mathrm{~h}$ ), as well as cerebellum from four further normal controls (two males and two females, age range (59-78 years), PMI between 59 and $78 \mathrm{~h}$ ) were included in the study. The Brain and Tissue Bank for Developmental Disorders (Maryland, USA) provided us with fresh frozen postmortem liver tissue from two individuals (one male and one female, both 18 years old, PMI 16 and $28 \mathrm{~h}$, respectively). Tissue was kept frozen at $-80^{\circ} \mathrm{C}$ until used.

\section{Selection of class specific oligonucleotide probes}

Oligonucleotides for in situ hybridization were designed using the online-program provided by the Alces Virtual Genome Center (http://alces.med.umn.edu/rawpeimer. $\mathrm{html})$. Probes that form hairpin formations were excluded by testing for possible RNA-folding using the MFOLD program (http://bioinfo.math.rpi.edu). All approved oligonucleotides were finally blasted against GenBank nonredundant and EST databases using parameters for identification of short nearly exact matches (http:// www.ncbi.nlm.nih.gov/BLAST/) in order to minimize unspecific binding to other mRNA species. All oligonucleotides (Table 2) were finally aligned pair-wise with mRNAs from the other classes to exclude those that are similar to other ADH classes. After this iterative process, for example our chosen rat class 3 ADHprobe (rADH3) does not show significant similarity to rat class I ADH or IV mRNAs or to any other rat mRNA as determined by the BLAST program.

\section{Oligonucleotide in situ hybridization}

The method used in this study is a modification of a previously published protocol [13]. In brief, unfixed cryosections of $14 \mu \mathrm{m}$ thickness were thawed onto coated glass slides (SuperFrost, VWR, Stockholm, Sweden) and kept at $-20{ }^{\circ} \mathrm{C}$ until use. Sections were removed from the freezer and air-dried $3-5 \mathrm{~h}$ prior to hybridization. Fifty nanomoles per slide of oligonucleotide probes (Table 2) were 3 '-endlabeled with $\left[\alpha-{ }^{33} \mathrm{P}\right] \mathrm{dATP}$ (NEN Lifescience, Boston, MA, USA) using terminal deoxynucleotidyl transferase (Amersham Pharmacia Biotech, Cleveland, OH, USA). Excess radioactive nucleotides were then removed (ProbeQuant G-50 Microcolumns, Amersham Pharmacia Biotech, Cleveland, $\mathrm{OH}, \mathrm{USA})$. Labeled oligonucleotide probes were diluted in hybridization cocktail containing $4 \times \mathrm{NaCl} / \mathrm{Cit}$, $50 \%$ formamide, $1 \times$ Denhardt's solution, $1 \%$ sarcosyl, 
Table 2. Sequences of the specific oligonucleotides used as in situ hybridization probes.

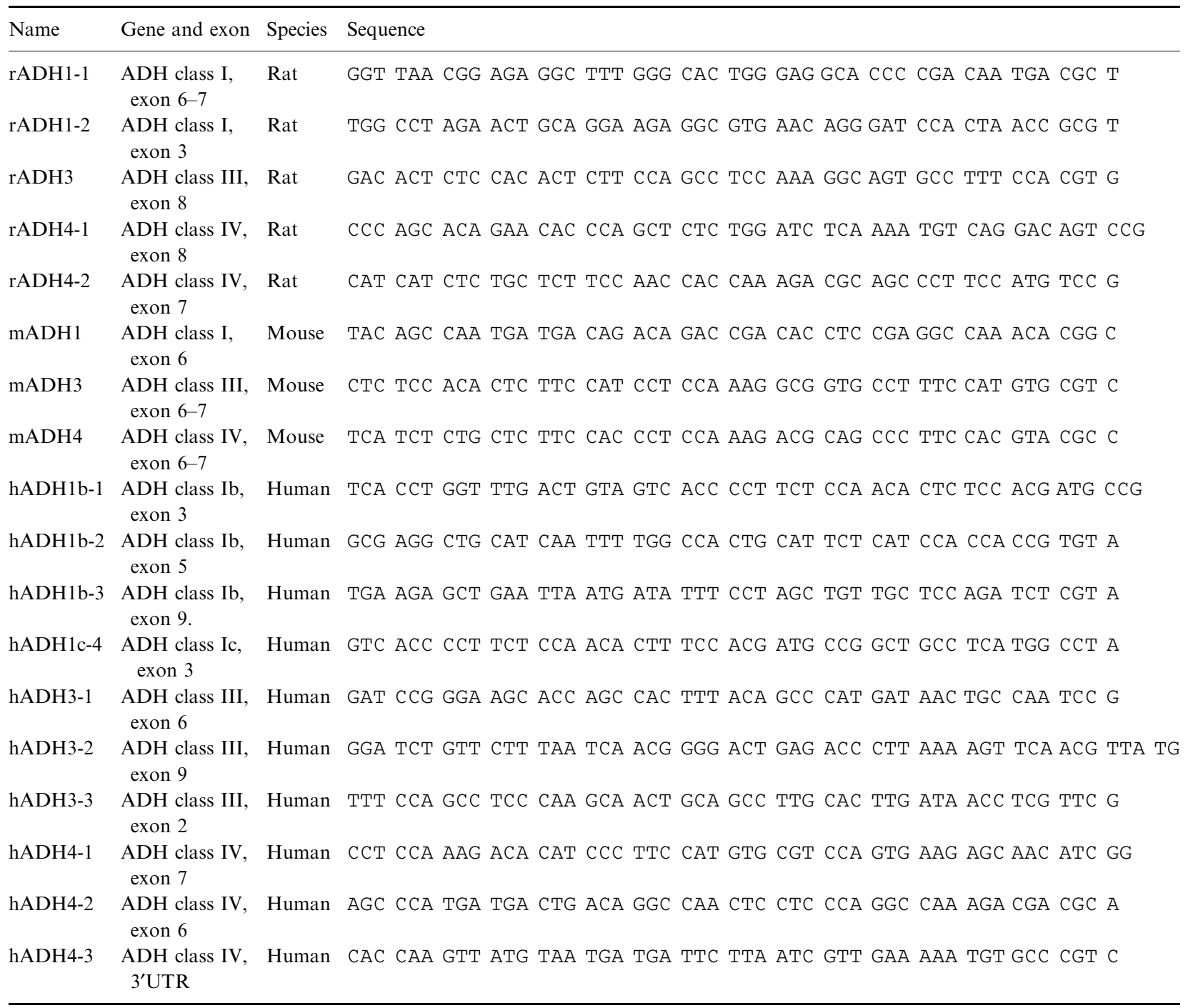

$0.02 \mathrm{~mol} \cdot \mathrm{L}^{-1}$ phosphate buffer $(\mathrm{pH}=7.0), 10 \%$ dextran sulfate, and $50 \mathrm{mg}$ sheared salmon sperm DNA, and $150 \mu \mathrm{L}$ of this solution was added to each slide followed by overnight incubation at $42{ }^{\circ} \mathrm{C}$ in a humidified chamber. After hybridization, slides were rinsed five times for $45 \mathrm{~min}$ at $60{ }^{\circ} \mathrm{C}$ in $1 \times \mathrm{NaCl} / \mathrm{Cit}$, rinsed once in water, dehydrated and air-dried. Slides were analyzed by phosphoimaging (FUJIX BAS 3000 system, Fujicolor Sweden AB, Skärholmen, Sweden) followed by dipping in photographic emulsion (Kodak NTB2 at 1:2 dilution, Kodak, Rochester, NY, USA). After exposure in the dark for three weeks, slides were developed, counterstained with cresyl violet and analyzed at the cellular level by dark- and brightfield microscopy. Material from at least two different rounds of in situ hybridization was analyzed for each probe by two independent observers. For rat tissue, we used two different probes for ADH1 and two for ADH4. For human tissue we used three different $\mathrm{ADH}$ probes for each of the three human ADH classes analyzed (see Table 2). We found similar expression patterns for all oligonucleotides designed for each class. Additionally, a random probe was used as negative control (data not shown).
Microphotographs were scanned, digitally processed and compiled using computer imaging software (Adobe PHOTOSHOP 5.5 and Adobe ILLUSTRATOR 8.0). Occasional particles of dust and other obvious artifacts were digitally retouched. Included microphotographs showing human tissue are high-power bright-field pictures, allowing silver grains in the photographic emulsion to be distinguished readily from neuromelanin or lipofuscin pigments abundantly present in human brain tissue.

\section{Results}

\section{Expression of different ADH classes in tissues outside the CNS}

Figures 1 and 2 show results from specificity tests of all probes on non-neuronal tissue (liver and stomach) where distributions of different ADH mRNA and protein species have been described previously. Both ADH1 and ADH3 were found to be expressed in liver (Figs 1A,C,E,G and $2 \mathrm{~B}, \mathrm{C}, \mathrm{F}, \mathrm{G})$, the tissue from which they were first purified and characterized $[14,15]$. 


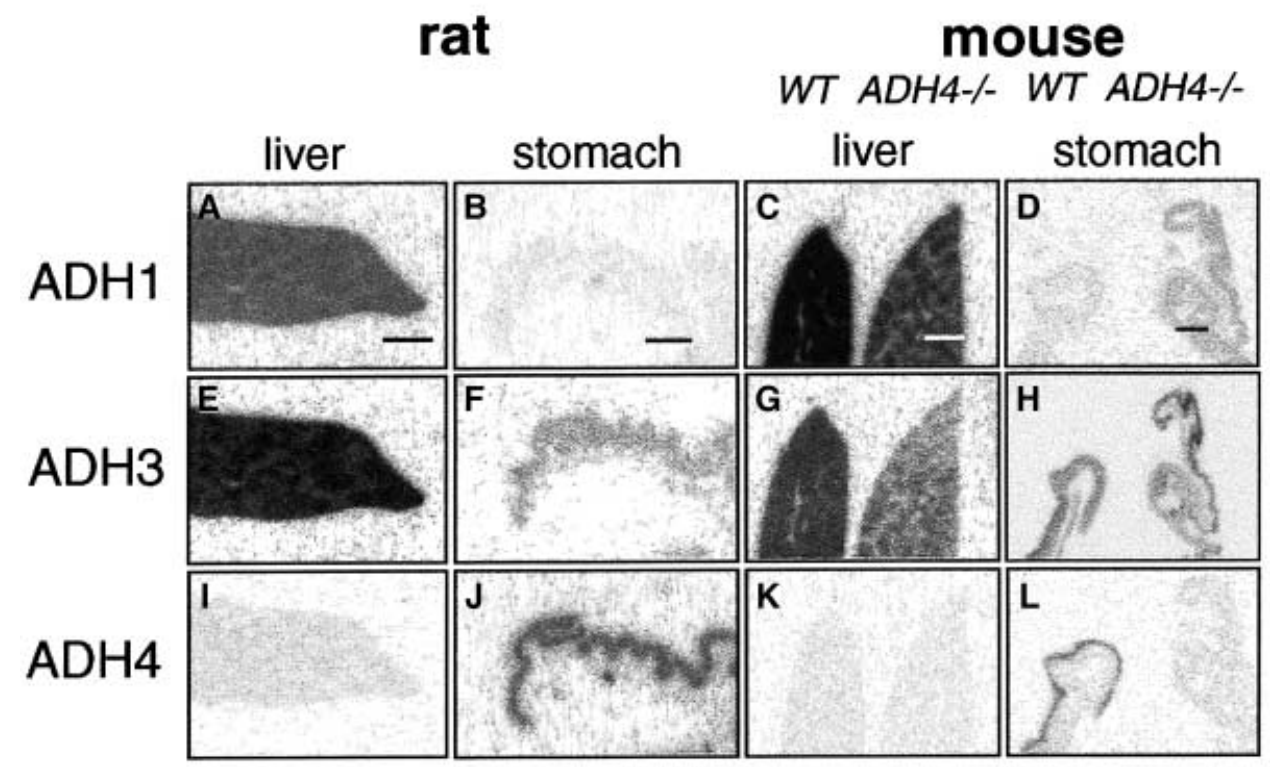

Fig. 1. Phosphoimager pictures of ADH class specific in situ hybridization signals from the indicated probes on control tissue from rat (A,B,E,F,I,J) and wild-type (WT, C,G,K) and ADH4 knock out (ADH4-/-, D,H,L) mice. Liver tissue expresses both ADH1 (A,C) and ADH3 (E,G), whereas ADH4 is expressed only in the stomach epithelium (J,L). Scale bar, $1.25 \mathrm{~mm}$.

High ADH1 mRNA expression levels were found in mouse (Fig. 1C) and human liver (Fig. 2B) and moderate expression levels in rat liver (Fig. 1A), in accordance with the literature [16-18]. The difference in the expression levels in the liver of the ADH4-/- and wild-type mice (Fig. 1C) might indicate that the transgenic manipulation at the ADH4 locus may actually affect expression levels at the nearby ADH1 locus located immediately downstream on chromosome 3 [19].

In rats, particularly high $\mathrm{ADH} 3$ expression was found in liver in accordance with studies showing that enzyme activity of ADH3 is highest in liver lysates [20], and immunostaining proving high protein expression in rat liver and colon [21].

ADH4, known as the stomach $\mathrm{ADH}$, was found not to be expressed in liver tissue in any of the three species (Figs 1I,K and 2D), as was expected from the literature $[22,23]$. ADH4 was strongly expressed in stomach epithelia of wild-type rodents, particularly of rats (Fig. 1J), while no signal was detectable in stomach epithelia of Adh4-/- mice (Fig. 1L) [12].

In mice and, predominantly, in rats, the deeper stomach epithelia also showed ADH3 expression (Figs 1F,H and $2 \mathrm{~K}$ ), a finding that has been reported previously by Northern blot analyses in humans [24] and rodents [17], by enzyme activity in human [9], and by immunohistochemistry also in rodents [22].

\section{Expression of ADH3 in the adult rodent and human brain}

Overviews of the expression patterns of the three classes of ADHs in the adult rodent brain are shown in Fig. 3 (scanned from phosphoimager plates). Coronary sections at three different levels were analyzed: forebrain (with anterior hippocampus), midbrain (including substantia nigra) and medulla oblongata with cerebellum.

Adh1 and Adh4 signals were absent in brain tissue from both rats and mice. Strong signal indicating high levels of ADH3 expression was present in the hippocampal formation and in cerebellum, weaker signal was detected in cortex cerebri.

To analyze the localization of $\mathrm{ADH}$ at the cellular level, slides were dipped in photographic emulsion, developed and analyzed under the microscope. Dark-field photomicrographs (Fig. 4) show the distribution of silver grains indicating expression in three regions of the rat brain. In hippocampus, ADH3 hybridization was strong within cornu amonis as well as in the dentate gyrus. In cortex, deeper layers gave rise to strong signals while upper layers showed only scattered expression and white matter showed no specific signal. In cerebellum, ADH3 hybridization was found in cells of the granular layer, the Purkinje cell layer and scattered areas of the molecular layer. A signal observed in cerebellar white matter with the ADH4 probe turned out to be unspecific: silver grains were not confined to cells and were present also in white matter of ADH4-/- mouse cerebellum, while the same probe did not give any signal in stomach tissue from this animal.

Figure 5 shows a bright-field view at higher magnification of ADH3 expression in rat brain. Many but not all neurons in cortex were ADH3 positive and all cerebellar Purkinje cells were strongly positive. Expression in the granular layer of rat and mouse cerebellum was moderate.

In hippocampus, neurons in the hilus of the dentate gyrus and granule cells of gyrus dentatus were clearly positive.

Hybridization of probes to choroid plexus tissue gave rise to a strong signal for ADH3 mRNA but no specific signal 

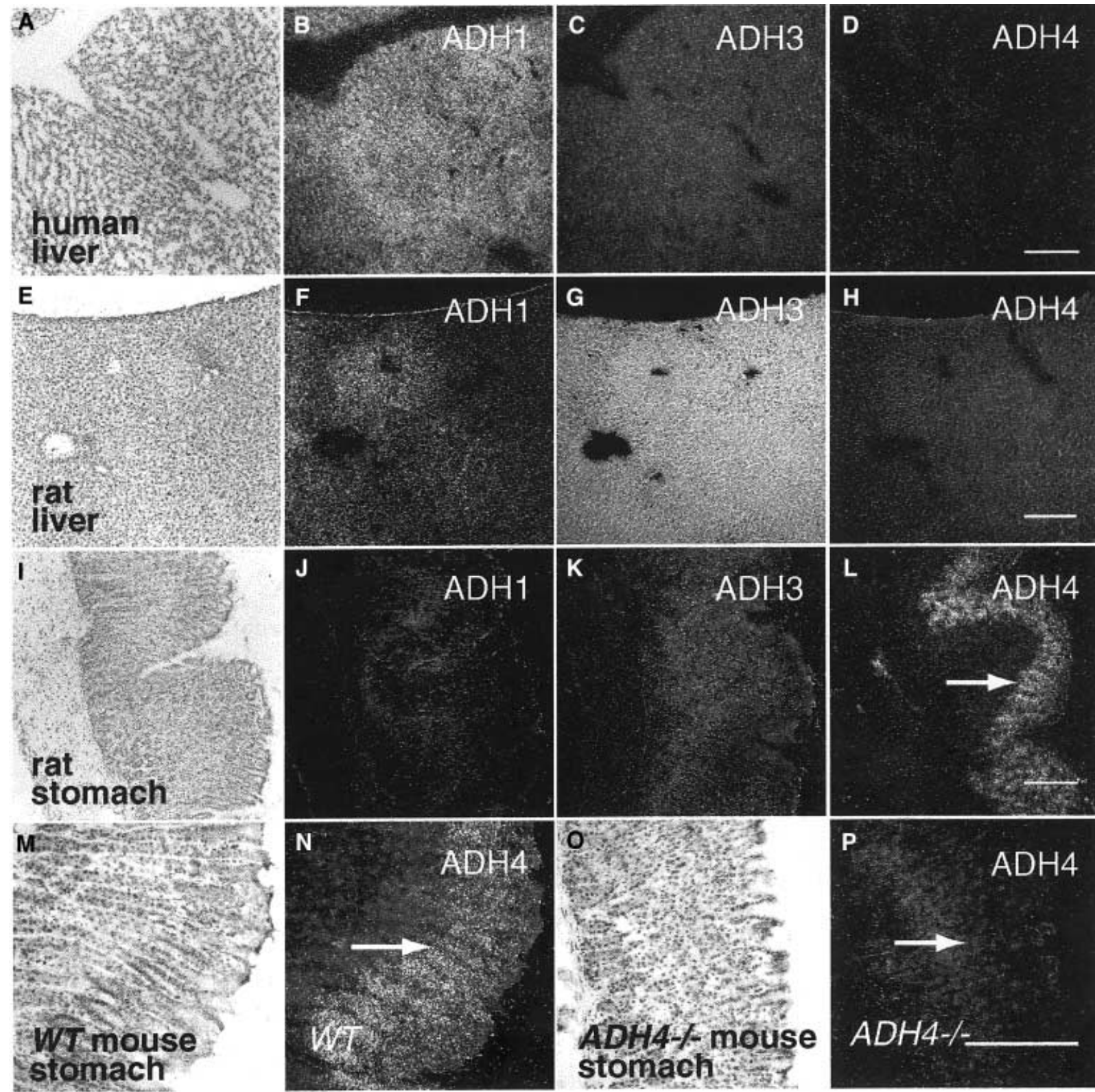

Fig. 2. Bright- and dark-field micrographs showing ADH mRNA signals in tissue outside of the CNS. In human liver (A-D) ADH1 is highly expressed (B), whereas in rat liver (E-H) ADH1 and ADH3 are both strongly expressed (F, G). The stomach epithelium of rats (I-L) shows specific expression of ADH4 (arrow, L) and wild-type mouse (N) but not in the ADH4-/- mouse (P). Scale bar, $500 \mu \mathrm{m}$.

for ADH4 in rats and mice. Figure 6 displays this finding in choroid plexus of the fourth ventricle in rat.

Comparisons of cellular expression patterns of ADHs between rodent and human brain revealed very similar results. As for rodent tissue, the only ADH detectable in cells of adult human brain was ADH3. Figure 7 shows several different regions in the human brain where ADH3 mRNA was detected: pyramidal neurons in cortex cerebri, CA3 pyramidal neurons as well as dopamine neurons of substantia nigra. A particularly strong ADH3 signal was detected in neurons of the human red nucleus. One finding that differed markedly between the species was absence of ADH3 mRNA in the granular layer of human cerebellum. Table 3 compiles our findings in adult brain tissue of all three species.

\section{Discussion}

The distribution of ADHs in the brain is of particular interest because of their implications in the metabolism of ethanol and retinoic acid. In vitro and in vivo data indicate that $\mathrm{ADH} 1, \mathrm{ADH} 3$ and $\mathrm{ADH} 4$ can oxidize retinol to retinal, with ADH4 having very high efficiency and ADH3 low efficiency [12,25,26]. All of these enzymes also metabolize ethanol with ADH1 having very high substrate affinity and ADH3 very low affinity [25]. The previously reported presence of $\mathrm{ADH} 1$ in the adult brain [11] might have been important with respect to ethanol abuse. However, our present results suggest that neither ADH1 nor ADH4 play key roles in brain ethanol metabolism, 


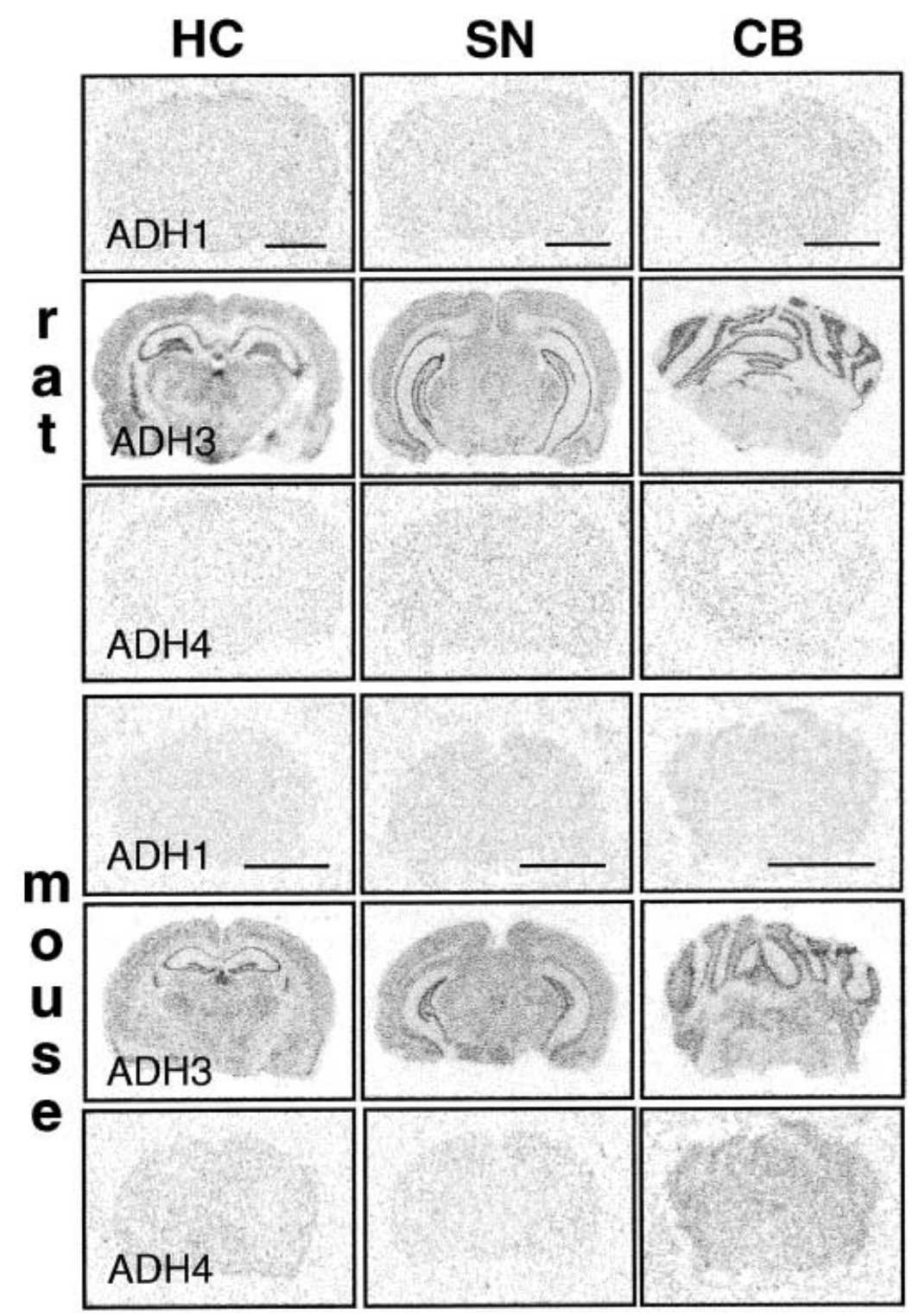

Fig. 3. Expression patterns of the different classes of ADH in the brains of rat and mouse at three different levels: hippocampus (HC), midbrain including substantia nigra (SN) and cerebellum (CB). Note that only AHD3 shows specific signals in the brains of both rat and mouse. Scale bars, $5 \mathrm{~mm}$.

but leave open the possibility that ADH3 might play a role in regions where it is expressed at high levels. Recently, it became apparent that ethanol can be oxidized in brain homogenates and that catalase is involved in the accumulation of acetaldehyde in the brain [27], explaining its presence despite the absence of ADH1 and the fact that acetaldehyde does not easily cross the blood brain barrier [28]. Although the accumulation of acetaldehyde has been proposed to contribute to addictive properties of alcohol [29], other studies suggest that accumulation of acetaldehyde may inhibit the drinking behavior due to uncomfortable feelings. In fact, increase of acetaldehyde levels by disulfiram, an inhibitor of the mitochondrial aldehyde dehyderogenase, is therapeutically used to deter alcohol drinking [30].
Retinoic acid has been implicated in many important functions during development, including development of the brain [31,32]. Accordingly, Adh4 expression has been detected in the embryonic mibrain floor [7,33]. Retinol is converted by this enzyme to retinal, that is further oxidized to retinoic acid by aldehyde dehydrogenase, an enzyme expressed specifically in dopamine neurons of substantia nigra [34]. Furthermore, a remarkable number of proteins involved in retinoid-related metabolism (retinoic acid receptors, cellular binding proteins and oxidizing enzymes) have been mapped within the adult dopamine system [35]. In the adult brain, retinoic acid has been proposed to be involved in synaptic plasticity [36,37] and neurogenesis [38].

The data that have been published concerning the localization and activity of the different $\mathrm{ADH}$ classes in 


\section{hippocampus}
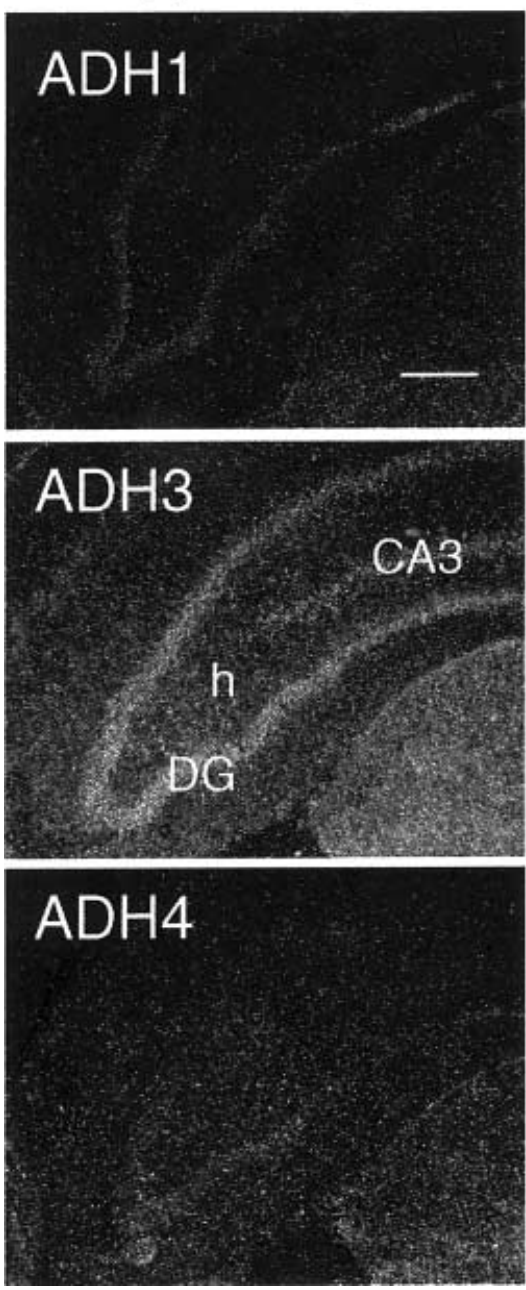

cortex
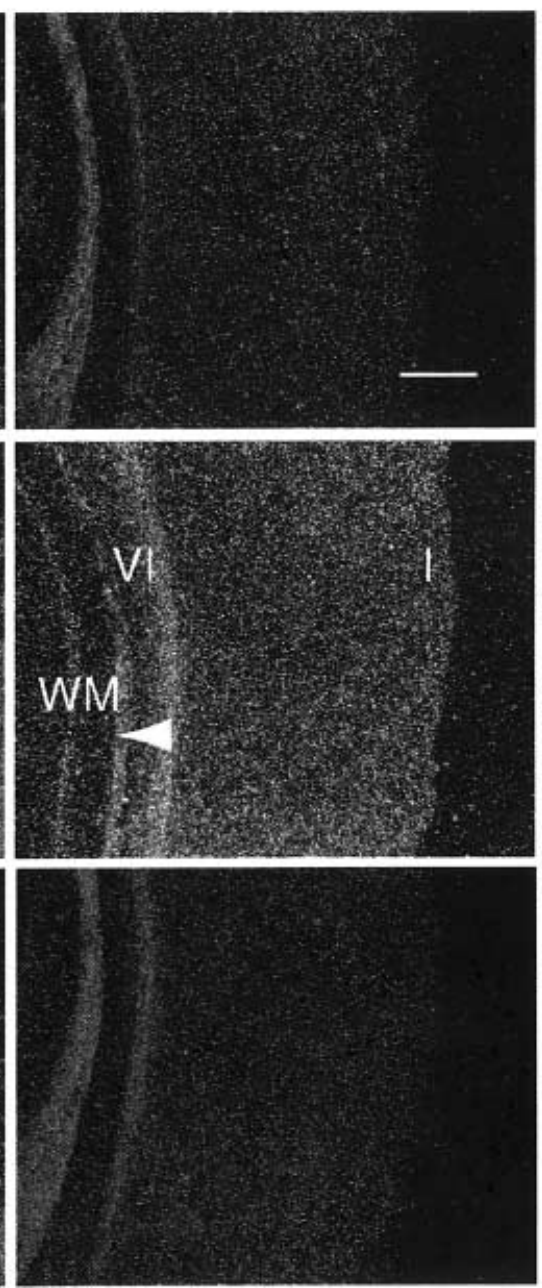

cerebellum
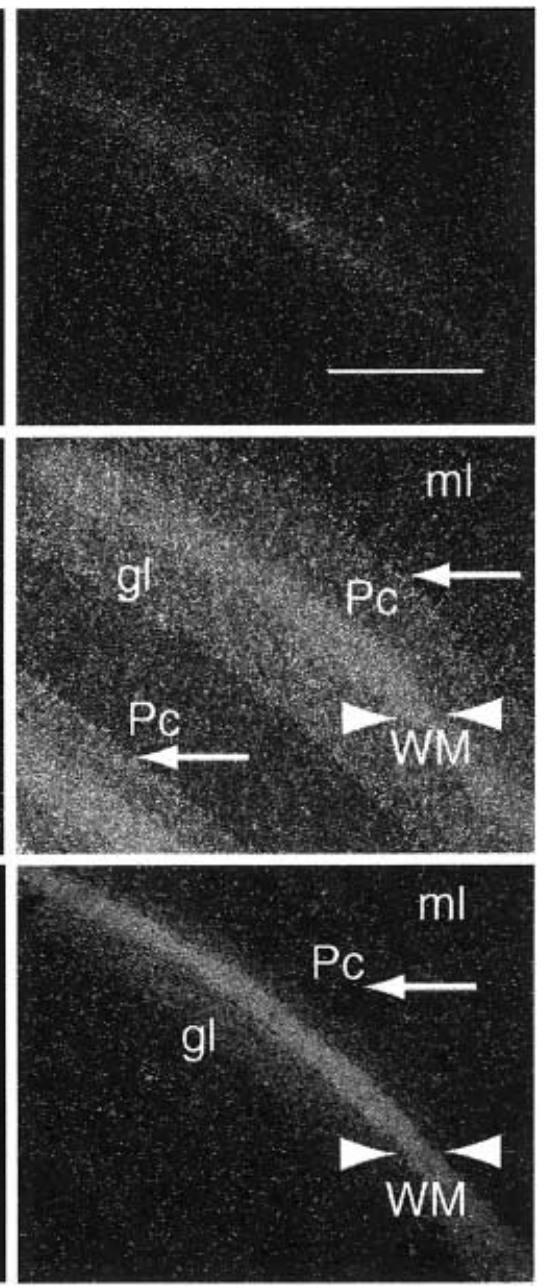

Fig. 4. Dark field micrographs showing the expression of ADH mRNA in the rat brain: cells in the dentate gyrus (DG), the hilus and the cornu amonis fields (CA) of hippocampus express only ADH3. In cortex, ADH3 mRNA was detected in scattered cells in the upper layer and in many cells in the lower layers, but not in white matter (WM). In cerebellum, Purkinje cells (Pc arrows), the granule cell layer (gl) and some cells in the molecular layer (ml) express ADH3 mRNA but no ADH4 or ADH1. The signal in cerebellar white matter with the ADH4 probe (arrowheads) is unspecific (see text). Scale bars, $500 \mu \mathrm{m}$.

the brain are partially contradictory. Our study supports the notion put forward in several studies that only class III ADH is expressed in the adult brain. Thus, Northern blot analysis from human brain homogenates had identified $\mathrm{ADH} 3$ as the only brain isoenzyme [5,6,18]. Another recent study investigated $\mathrm{ADH} 1$ and $\mathrm{ADH} 3$ protein expression in human brain by Western blot analysis and immunohistochemistry [39]. By this methodology, highest ADH3 protein levels were found in cerebellum and hippocampus, and lower levels in different regions of cortex cerebri. The cellular distribution of ADH3 protein coincides with our findings of mRNA: cortical neurons in deeper layers, hippocampal neurons and Purkinje cells of cerebellum. Additionally, notably high expression levels of $\mathrm{ADH} 3$ in nucleus ruber were identified in the present study, a finding that has not been described before. In the above-cited study, as well as the present work, ADH1 expression was not detected in any of the investigated brain regions.

Expression of ADH1 and ADH4 mRNA in the rat adult brain has been claimed by one study using partially hydrolyzed riboprobe in situ hybridization [11]. The authors found ADH1 expression in cerebellar granule cells and Purkinje cells, in the hippocampal formation and different regions of the cerebral cortex. ADH4 mRNA expression was found in Purkinje cells and white matter of the cerebellum, and in hippocamus and cortex. Both ADH classes were also shown to be present in the choroid plexus. These data are in contradiction with our findings, as we found expression of $\mathrm{ADH} 3$ in all these cell types but neither ADH1 nor ADH4. One explanation for this discrepancy may be that the oligonucleotides used in the present study may not have been sensitive enough to detect possible low expression levels of these enzyme classes. Based on the 
Fig. 5. ADH3 expression in neurons from different regions of the rat brain: pyramidal cells of the cortex and in the hilus of the dentate gyrus, Purkinje cells and granule cells in the cerebellum. Scale bar, $45 \mu \mathrm{m}$.
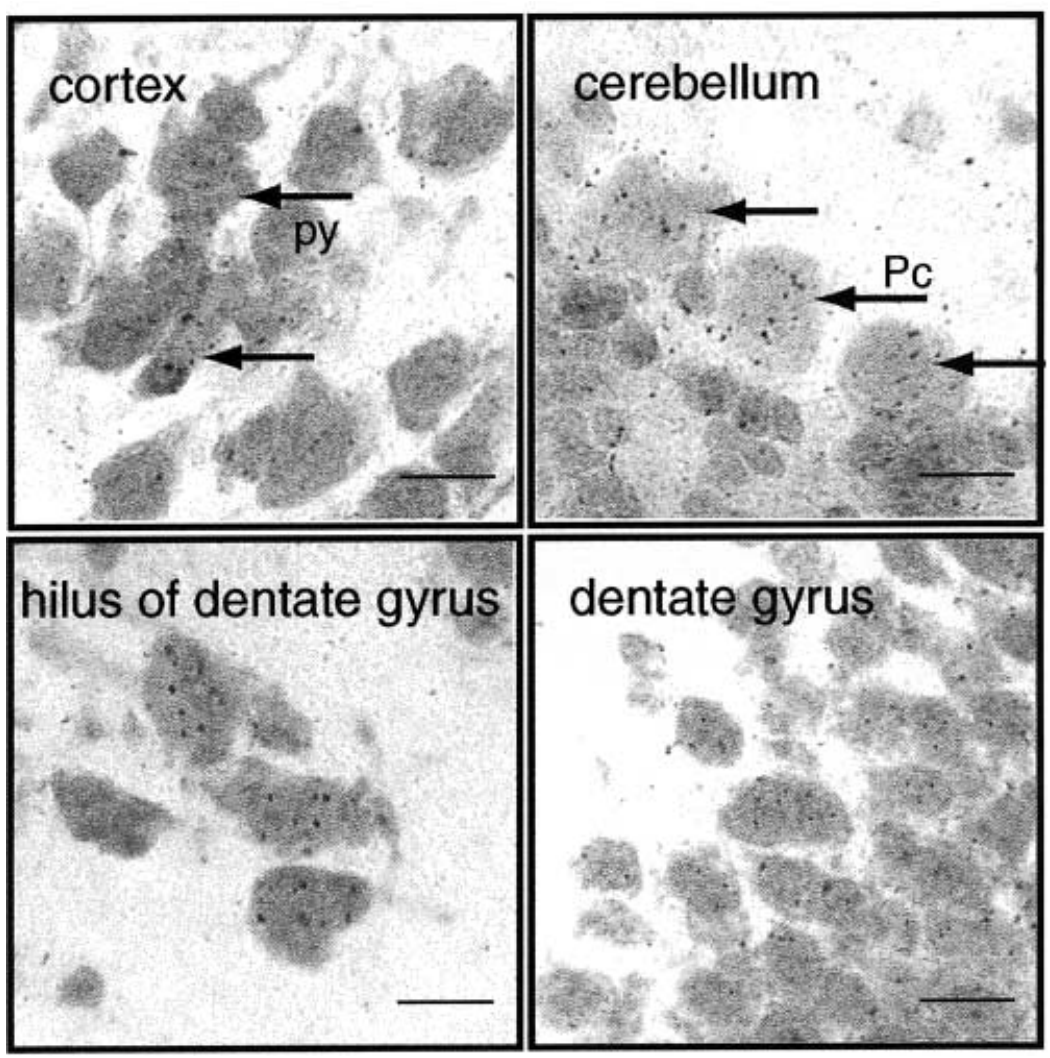
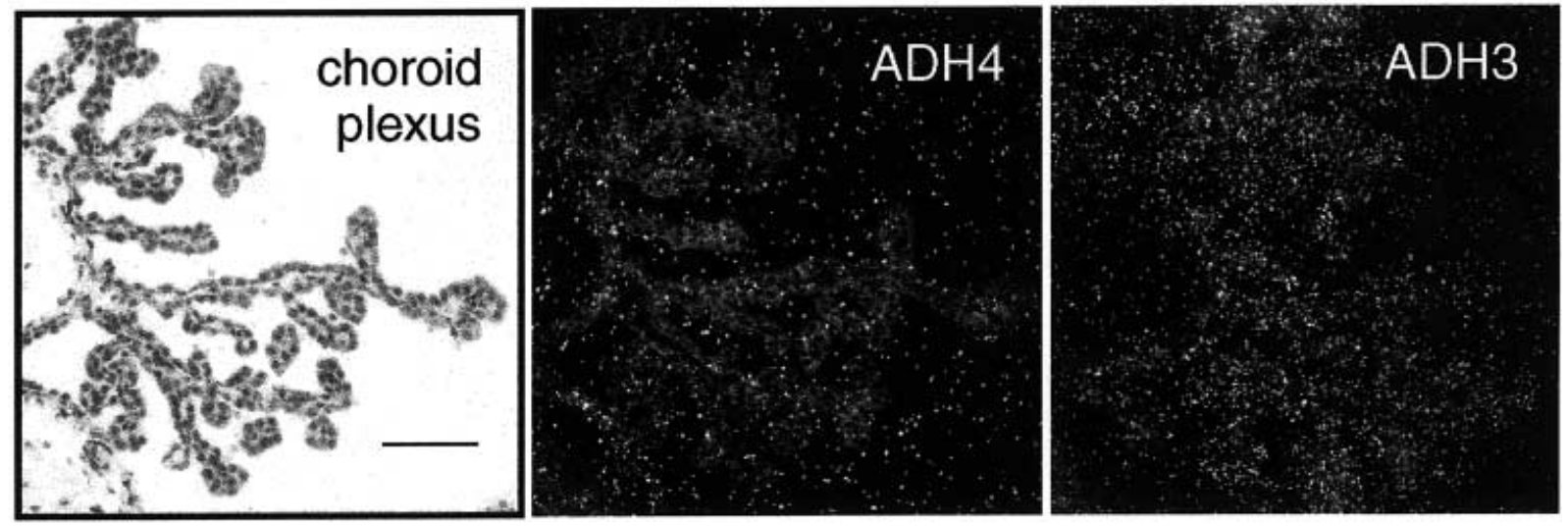

Fig. 6. In situ hybridization showing an ADH3 specific signal in the choroid plexus of the fourth ventricle of the rat brain, but no ADH4 specific signal. Scale bar, $150 \mu \mathrm{m}$.

above-described patterns of signals, however, it appears more likely that the discrepancy is due to insufficient specificity of the hydrolyzed riboprobes leading to crossreactions of the ADH1 and $\mathrm{ADH} 4$ probes with the orthologous ADH3 mRNA. Martinez et al. [11] point out that their study is in agreement with findings from an immunohistochemical study localizing $\mathrm{ADH}$ in the rat brain [40]. The antibody used in this study was raised against isolated rat liver $\mathrm{ADH}$, without any further characterization concerning the class specificity. As rat liver expresses both
ADH1 and, very strongly, $\mathrm{ADH} 3$, such immunohistochemistry results can however, not differentiate between $\mathrm{ADH} 1$ and $\mathrm{ADH} 3$. Our results are also in agreement with the finding that only $\mathrm{ADH} 3$ activity is detectable in homogenates of different parts of the rat brain in starch gel electrophoresis followed by ADH activity staining [11].

In mice, $\mathrm{ADH}$ expression in the adult brains of 15 different inbred strains has been investigated by isoelectric focusing followed by staining of enzyme activity [41]. ADH3 activity was detected in all strains studied whereas ADH1 


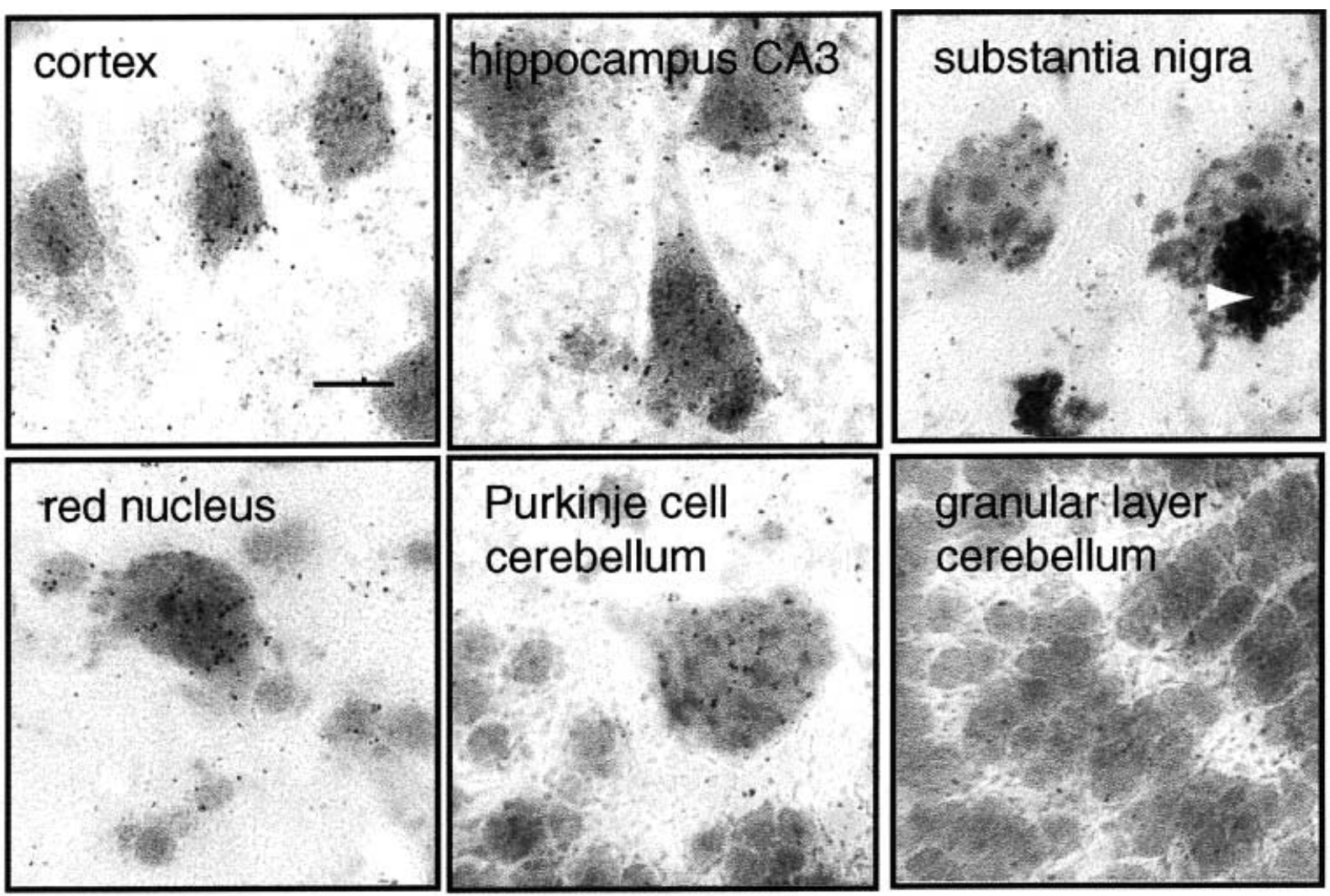

Fig. 7. ADH3 expression in neurons from different regions of the human brain: pyramidal neurons in cortex cerebri and the CA3 region of hippocampus, dopamine neurons in substantia nigra pars compacta (arrowhead indicates neuromelanin granules in one of the two cell bodies), magnocellular neurons in the red nucleus and Purkinje cells in cerebellum. Neurons in the granular layer of cerebellum do not express ADH3. Scale bar, $45 \mu \mathrm{m}$.

Table 3. Distribution of the three ADH classes in adult rat, mouse and human brain tissue. The presence of specific signal is shown by + , strong presence by ++ and absence by - .

\begin{tabular}{|c|c|c|c|c|c|c|c|c|c|}
\hline \multirow[b]{2}{*}{ Brain area } & \multicolumn{3}{|l|}{ Rat } & \multicolumn{3}{|l|}{ Mouse } & \multicolumn{3}{|l|}{ Human } \\
\hline & ADH1 & $\mathrm{ADH} 3$ & ADH4 & $\mathrm{ADH} 1$ & $\mathrm{ADH} 3$ & $\mathrm{ADH} 4$ & ADH1 & $\mathrm{ADH} 3$ & $\mathrm{ADH} 4$ \\
\hline \multicolumn{10}{|l|}{ Cortex } \\
\hline Retrosplenial agranular cortex & - & - & - & - & - & - & $-{ }^{\mathrm{a}}$ & $++^{\mathrm{a}}$ & $-{ }^{\mathrm{a}}$ \\
\hline Retrosplenial granular cortex & - & + & - & - & + & - & & & \\
\hline Visual cortex & - & + & - & - & + & - & & & \\
\hline Viriform cortex & - & + & - & - & + & - & & & \\
\hline White matter & - & - & - & - & - & - & - & - & - \\
\hline \multicolumn{10}{|l|}{ Hippocampal formation } \\
\hline Dentate gyrus & - & + & - & - & + & - & - & + & - \\
\hline Hilus & - & + & - & - & + & - & - & + & - \\
\hline CA1-3 & - & + & - & - & ++ & - & - & $++^{b}$ & - \\
\hline \multicolumn{10}{|l|}{ Midbrain } \\
\hline Substantia nigra & - & + & - & - & + & - & - & + & - \\
\hline Red nucleus & - & + & - & - & + & - & - & ++ & - \\
\hline Locus coeruleus & - & + & - & - & + & - & - & + & - \\
\hline \multicolumn{10}{|l|}{ Cerebellum } \\
\hline Granular layer & - & + & - & - & + & - & - & - & - \\
\hline Purkinje cells & - & ++ & - & - & ++ & - & - & ++ & - \\
\hline Molecular layer & - & + & - & - & + & - & - & + & - \\
\hline White matter & - & - & - & - & - & $-{ }^{c}$ & - & - & - \\
\hline Choroid plexus & - & + & - & - & + & - & - & + & - \\
\hline
\end{tabular}

${ }^{\mathrm{a}}$ Frontal cortex, ${ }^{\mathrm{b}}$ only CA3 studied, ${ }^{\mathrm{c}}$ unspecific signal, present in ADH4-/- as well. 
activity was very low or absent in the investigated brain extracts. These data support our findings in mice.

Regarding the apparent ADH4 expression in cerebellar white matter that has been reported by the same authors [11] and that we also observed in both rat and mice, we have now shown that it must be due to unspecific stickiness of the probes because it was present even in the ADH4 knock-out mice that had been shown previously to completely lack ADH4 mRNA due to deletion of the promoter [12]. In these mice, we could clearly demonstrate absence of Adh4 mRNA in the stomach epithelia - the tissue with the best characterized Adh4 expression.

Taken together, our results demonstrate expression of ADH3 in most of the analyzed areas in the brain, with highest expression levels in hippocampus, cerebellum and particularly in human brain, in the red nucleus. Using the same methodology, no ADH1 or ADH4 expression was detected. The only clear difference between the species we detected in the brain was in cerebellum, where the granular layer expresses Adh3 in rodents but not in humans. The relative abundance of $\mathrm{ADH} 3$ within many different tissue types is probably related to the need of scavenging formaldehyde for cytoprotection, but low activity of ADH3 with ethanol and retinol cannot be ruled out. Our results do not support a significant involvement of $\mathrm{ADH} 1$ and ADH4 in ethanol oxidation in brain tissue. Regarding retinoid metabolism in the adult brain, enzymes other than ADH4 must be active, because in vivo and in vitro data indicate that adult brain tissue, in particularly the striatum, can oxidize retinol to retinal, providing the first step on the way to retinoic acid [31]. The brain must thus rely on the activity of other enzymes, for example ADH3 or other members of the medium-chain dehydrogenase/reductase family (MDR), or perhaps members of the short-chain dehydrogenases/reductase family (SDR), both of which utilize a variety of metabolites and toxic compounds [42].

\section{Acknowledgements}

Human brain tissue samples were provided by the Harvard Brain Tissue Resource Center that is supported in part by grant number $\mathrm{MH} /$ NS 31862. We acknowledge the NIH and the Brain and Tissue Bank for Developmental Disorders, that is supported in part by grant number N01-HD-1-3138, for the human liver tissue samples. We thank Karin Lundströmer, Karin Pernold and Eva Lindqvist for technical assistance. Supported by the Swedish Research Council, the Swedish Parkinson Foundation, Karolinska Institutet funds, Deutsche Forschungsgemeinschaft (DFG) grant GA 2/1 and National Institutes of Health grant AA09731.

\section{References}

1. Jörnvall, H. (1994) The alcohol dehydrogenase system. EXS 71, 221-229.

2. Duester, G., Farres, J., Felder, M.R., Holmes, R.S., Höög, J.O., Pares, X., Plapp, B.V., Yin, S.J. \& Jörnvall, H. (1999) Recommended nomenclature for the vertebrate alcohol dehydrogenase gene family. Biochem. Pharmacol. 58, 389-395.

3. Danielsson, O. \& Jörnvall, H. (1992) 'Enzymogenesis': classical liver alcohol dehydrogenase origin from the glutathione-dependent formaldehyde dehydrogenase line. Proc. Natl Acad. Sci. USA 89, 9247-9251.
4. Nordling, E., Persson, B. \& Jörnvall, H. (2002) Differential multiplicity of MDR alcohol dehydrogenases: enzyme genes in the human genome versus those in organisms initially studied. Cell Mol. Life Sci. 59, 1070-1075.

5. Giri, P.R., Linnoila, M., O’Neill, J.B. \& Goldman, D. (1989) Distribution and possible metabolic role of class III alcohol dehydrogenase in the human brain. Brain Res. 481, 131-141.

6. Beisswenger, T.B., Holmquist, B. \& Vallee, B.L. (1985) chi-ADH is the sole alcohol dehydrogenase isozyme of mammalian brains: implications and inferences. Proc. Natl Acad. Sci. USA 82, 8369-8373.

7. Haselbeck, R.J. \& Duester, G. (1998) ADH4-lacZ. transgenic mouse reveals alcohol dehydrogenase localization in embryonic midbrain/hindbrain, otic vesicles, and mesencephalic, trigeminal, facial, and olfactory neural crest. Alcohol Clin. Exp. Res. 22, 1607-1613.

8. Saleem, M.M., al Tamer, Y.Y., Skursky, L. \& Al Habbal, Z. (1984) Alcohol dehydrogenase activity in the human tissues. Biochem. Med. 31, 1-9.

9. Jelski, W., Chrostek, L., Szmitkowski, M. \& Laszewicz, W. (2002) Activity of class I, II, III, and IV alcohol dehydrogenase isoenzymes in human gastric mucosa. Dig. Dis. Sci. 47, 1554-1557.

10. Matsumoto, M., Yokoyama, H., Shiraishi, H., Suzuki, H., Kato, S., Miura, S. \& Ishii, H. (2001) Alcohol dehydrogenase activities in the human gastric mucosa: Effects of Helicobacter pylori infection, sex, age, and the part of the stomach. Alcoholism-Clin. Exp Res. 25, 29S-34S.

11. Martinez, S.E., Vaglenova, J., Sabria, J., Martinez, M.C., Farres, J. \& Pares, X. (2001) Distribution of alcohol dehydrogenase mRNA in the rat central nervous system. Consequences for brain ethanol and retinoid metabolism. Eur. J. Biochem. 268, 5045-5056.

12. Deltour, L., Foglio, M.H. \& Duester, G. (1999) Impaired retinol utilization in Adh4 alcohol dehydrogenase mutant mice. Dev. Genet. 25, 1-10.

13. Dagerlind, A., Friberg, K., Bean, A.J. \& Hökfelt, T. (1992) Sensitive mRNA detection using unfixed tissue: combined radioactive and non-radioactive in situ hybridization histochemistry. Histochemistry 98, 39-49.

14. Uotila, L. \& Koivusalo, M. (1974) Formaldehyde dehydrogenase from human liver. Purification, properties, and evidence for the formation of glutathione thiol esters by the enzyme. J. Biol. Chem. 249, 7653-7663.

15. von Wartburg, J.P., Bethune, J.L. \& Vallee, B.L. (1964) Human liver-alcohol dehydrogenase. Kinetic and physicochemical properties. Biochemistry 3, 1775-1782.

16. Ang, H.L., Deltour, L., Zgombic-Knight, M., Wagner, M.A. \& Duester, G. (1996) Expression patterns of class I and class IV alcohol dehydrogenase genes in developing epithelia suggest a role for alcohol dehydrogenase in local retinoic acid synthesis. Alcohol Clin. Exp. Res. 20, 1050-1064.

17. Estonius, M., Danielsson, O., Höög, J.O., Persson, H. \& Jörnvall, H. (1993) Tissue distribution of alcohol and sorbitol dehydrogenase mRNAs. Adv. Exp. Med. Biol. 328, 545-553.

18. Estonius, M., Svensson, S. \& Höög, J.O. (1996) Alcohol dehydrogenase in human tissues: localisation of transcripts coding for five classes of the enzyme. FEBS Lett. 397, 338-342.

19. Szalai, G., Duester, G., Friedman, R., Jia, H., Lin, S., Roe, B.A. \& Felder, M.R. (2002) Organization of six functional mouse alcohol dehydrogenase genes on two overlapping bacterial artificial chromosomes. Eur. J. Biochem. 269, 224-232.

20. Casanova-Schmitz, M., David, R.M. \& Heck, H.D. (1984) Oxidation of formaldehyde and acetaldehyde by NAD +dependent dehydrogenases in rat nasal mucosal homogenates. Biochem. Pharmacol. 33, 1137-1142. 
21. Keller, D.A., Heck, H.D., Randall, H.W. \& Morgan, K.T. (1990) Histochemical localization of formaldehyde dehydrogenase in the rat. Toxicol. Appl. Pharmacol. 106, 311-326.

22. Haselbeck, R.J. \& Duester, G. (1997) Regional restriction of alcohol/retinol dehydrogenases along the mouse gastrointestinal epithelium. Alcohol Clin. Exp. Res. 21, 1484-1490.

23. Zgombic-Knight, M., Foglio, M.H. \& Duester, G. (1995) Genomic structure and expression of the $\mathrm{ADH} 7$ gene encoding human class IV alcohol dehydrogenase, the form most efficient for retinol metabolism in vitro. J. Biol. Chem. 270, 4305-4311.

24. Engeland, K. \& Maret, W. (1993) Extrahepatic, differential expression of four classes of human alcohol dehydrogenase. Biochem. Biophys. Res. Commun. 193, 47-53.

25. Boleda, M.D., Saubi, N., Farres, J. \& Pares, X. (1993) Physiological substrates for rat alcohol dehydrogenase classes: aldehydes of lipid peroxidation, omega-hydroxyfatty acids, and retinoids. Arch. Biochem. Biophys. 307, 85-90.

26. Molotkov, A., Fan, X., Deltour, L., Foglio, M.H., Martras, S., Farres, J., Pares, X. \& Duester, G. (2002) Stimulation of retinoic acid production and growth by ubiquitously expressed alcohol dehydrogenase Adh3. Proc. Natl Acad. Sci. USA 99, 5337-5342.

27. Person, R.E., Chen, H., Fantel, A.G. \& Juchau, M.R. (2000) Enzymic catalysis of the accumulation of acetaldehyde from ethanol in human prenatal cephalic tissues: evaluation of the relative contributions of CYP2E1, alcohol dehydrogenase, and catalase/peroxidases. Alcohol Clin. Exp. Res. 24, 1433-1442.

28. Zimatkin, S.M., Liopo, A.V. \& Deitrich, R.A. (1999) Oxidation of ethanol to acetaldehyde in brain and the possible behavioral consequences. Adv. Exp. Med. Biol. 463, 231-236.

29. McBride, W.J., Li, T.K., Deitrich, R.A., Zimatkin, S., Smith, B.R. \& Rodd-Henricks, Z.A. (2002) Involvement of acetaldehyde in alcohol addiction. Alcohol Clin. Exp. Res. 26, 114-119.

30. Johansson, B. (1992) A review of the pharmacokinetics and pharmacodynamics of disulfiram and its metabolites. Acta Psychiatr. Scand. Suppl. 369, 15-26.

31. McCaffery, P. \& Dräger, U.C. (2000) Regulation of retinoic acid signaling in the embryonic nervous system: a master differentiation factor. Cytokine Growth Factor Rev. 11, 233-249.
32. Zetterström, R.H., Lindqvist, E., Mata de Urquiza, A., Tomac, A., Eriksson, U., Perlmann, T. \& Olson, L. (1999) Role of retinoids in the CNS: differential expression of retinoid binding proteins and receptors and evidence for presence of retinoic acid. Eur. J. Neurosci. 11, 407-416.

33. Ang, H.L., Deltour, L., Hayamizu, T.F., Zgombic-Knight, M. \& Duester, G. (1996) Retinoic acid synthesis in mouse embryos during gastrulation and craniofacial development linked to class IV alcohol dehydrogenase gene expression. J. Biol. Chem. 271, 9526-9534.

34. McCaffery, P. \& Dräger, U.C. (1994) High levels of a retinoic acidgenerating dehydrogenase in the meso-telencephalic dopamine system. Proc. Natl Acad. Sci. USA 91, 7772-7776.

35. Zetterström, R.H., Simon, A., Giacobini, M.M., Eriksson, U. \& Olson, L. (1994) Localization of cellular retinoid-binding proteins suggests specific roles for retinoids in the adult central nervous system. Neuroscience 62, 899-918.

36. Misner, D.L., Jacobs, S., Shimizu, Y., de Urquiza, A.M., Solomin, L., Perlmann, T., De Luca, L.M., Stevens, C.F. \& Evans, R.M. (2001) Vitamin A deprivation results in reversible loss of hippocampal long-term synaptic plasticity. Proc. Natl Acad. Sci. USA 98, 11714-11719.

37. Thompson, H.G., Maynard, T.M., Shatzmiller, R.A. \& Lamantia, A.S. (2002) Retinoic acid signaling at sites of plasticity in the mature central nervous system. J. Comp Neurol. 452, 228-241.

38. Takahashi, J., Palmer, T.D. \& Gage, F.H. (1999) Retinoic acid and neurotrophins collaborate to regulate neurogenesis in adultderived neural stem cell cultures. J. Neurobiol. 38, 65-81.

39. Mori, O., Haseba, T., Kameyama, K., Shimizu, H., Kudoh, M., Ohaki, O., Arai, Y., Yamazaki, M. \& Asano, G. (2000) Histological distribution of class III alcohol dehydrogenase in human brain. Brain Res. 852, 186-190.

40. Kerr, J.T., Maxwell, D.S. \& Crabb, D.W. (1989) Immunocytochemistry of alcohol dehydrogenase in the rat central nervous system. Alcohol Clin. Exp. Res. 13, 730-736.

41. Rout, U.K. (1992) Alcohol dehydrogenases in the brain of mice. Alcohol Clin. Exp. Res. 16, 286-289.

42. Duester, G. (2000) Families of retinoid dehydrogenases regulating vitamin A function: production of visual pigment and retinoic acid. Eur. J. Biochem. 267, 4315-4324. 